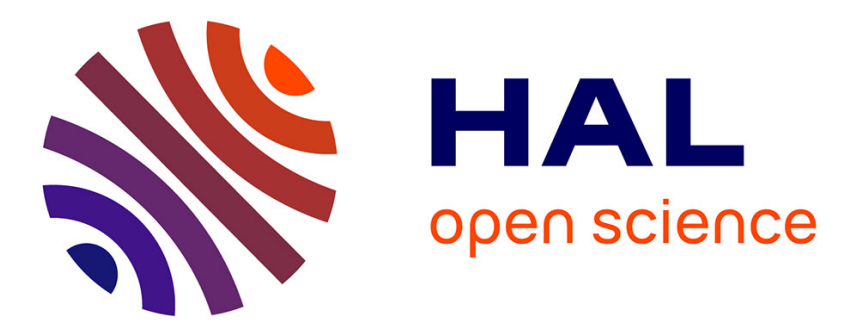

\title{
Evolution du complexe parasitaire constitué par Rhizoctonia cerealis Van der Hoeven et Pseudocercosporella herpotrichoides (Fron) Deighton, champignons parasites de la base des tiges de céréales
}

Nadine Cavelier, Philippe Lucas, Guénolé Boulch

\section{To cite this version:}

Nadine Cavelier, Philippe Lucas, Guénolé Boulch. Evolution du complexe parasitaire constitué par Rhizoctonia cerealis Van der Hoeven et Pseudocercosporella herpotrichoides (Fron) Deighton, champignons parasites de la base des tiges de céréales. Agronomie, 1985, 5 (8), pp.693-700. hal00884801

\section{HAL Id: hal-00884801 https://hal.science/hal-00884801}

Submitted on 1 Jan 1985

HAL is a multi-disciplinary open access archive for the deposit and dissemination of scientific research documents, whether they are published or not. The documents may come from teaching and research institutions in France or abroad, or from public or private research centers.
L'archive ouverte pluridisciplinaire HAL, est destinée au dépôt et à la diffusion de documents scientifiques de niveau recherche, publiés ou non, émanant des établissements d'enseignement et de recherche français ou étrangers, des laboratoires publics ou privés. 


\title{
Evolution du complexe parasitaire constitué par Rhizoctonia cerealis Van der Hoeven et Pseudo- cercosporella herpotrichoides (Fron) Deighton, champignons parasites de la base des tiges de céréales
}

Nadine CAVELIER $\left({ }^{*}\right)$, Philippe LUCAS \& Guénolé BOULCH

I.N.R.A., Station de Pathologie végétale, Centre de Recherches de Rennes, F 35650 Le Rheu

(*) S.R.I.V. : Service de recherches intégrées sur les productions végétales et la protection des plantes

RÉSUMÉ

\begin{abstract}
Si le piétin-verse dû à Pseudocercosporella herpotrichoides a longtemps été considéré, en France, comme la seule maladie importante de la base des tiges de céréales, le rhizoctone causé par Rhizoctonia cerealis semble aujourd'hui prendre de l'extension.

Des expérimentations sur blé tendre d'hiver sont menées avec ces deux parasites en conditions contrôlées et au champ afin de suivre leur évolution simultanée. Dans les deux cas, les observations sont faites après contamination artificielle.

En l'absence de traitement fongicide, la fréquence des symptômes dus à $R$. cerealis est moins importante quand ces deux champignons sont présents dans une même parcelle que lorsque $R$. cerealis s'y trouve seul. Il semblerait donc que $P$. herpotrichoides soit un parasite dominant.

Après traitement au carbendazime, la fréquence des symptômes provoqués par $\boldsymbol{P}$. herpotrichoides est fortement réduite, tandis que celle des symptômes dus à $R$. cerealis reste la même ou augmente.
\end{abstract}

Mots clés additionnels : Traitement fongicide, carbendazime, blé, rhizoctone, piétin-verse. Pseudocercosporella herpotrichoides (Fron) Deighton causing foot rot diseases of cereals.

In France, among the pathogens causing foot rot of cereals, $P$. herpotrichoides has always been the most important. During recent years, however, $R$. cerealis has become increasingly important.

In the presence of both pathogens, $P$. herpotrichoides was the predominant pathogen in untreated plots. After a carbendazim treatment, the frequency of eyespot symptoms decreased considerably, while that of sharp eyespot remained at the same level or increased.

In vitro, mean ED 50 values for carbendazim were $1.9 \mu \mathrm{g} / \mathrm{ml}$ for $R$. cerealis and $0.06 \mu \mathrm{g} / \mathrm{ml}$ for $P$. herpotrichoides.

Additional key words : fungicide treatments, carbendazim, wheat, sharp eyespot, eyespot.

\section{INTRODUCTION}

Le piétin-verse provoqué par Pseudocercosporella herpotrichoides (Fron) Deighton est une maladie grave et largement répandue qui a fait l'objet de nombreuses études tant en France qu'à l'étranger : SPRAGUE (1931) aux Etats-Unis, GLYNNE (1936) en Grande-
Bretagne, PONCHET (1959) en France, pour ne citer que quelques exemples.

Avec l'apparition des fongicides de la famille des benzimidazoles et thiophanates, une méthode efficace de lutte chimique a été mise au point : DEFOSSE (1970), LeMAIRE et al. (1970), FEHRMANN (1970) et RAPILLY (1970). 
DOUSSINAULT (1970) a orienté ses recherches sur la sélection de cultivars résistants au piétin-verse. Cellesci ont abouti à la création de la variété « Roazon » et à l'obtention d'autres géniteurs intéressants pour ce caractère.

Mais parallèlement à la mise en œuvre de ces méthodes de lutte, d'autres maladies semblent avoir pris de l'importance. C'est le cas des fusarioses et surtout du rhizoctone. Ce dernier, dû à Rhizoctonia cerealis Van der Hoeven, est de plus en plus observé en France depuis quelques années et, si sa nuisibilité n'a pas encore été parfaitement établie, sa spécificité sur céréales par rapport à $R$. solani a été démontrée (LUCAS \& CAVELIER, 1983).

Or, les applications de fongicides en cours de végétation sont en constante progression depuis 1973 et l'utilisation de spécialités à base de benzimidazoles ou thiophanates (bénomyl, carbendazime, méthylthiophanate) contre le piétin-verse préoccupe plusieurs auteurs, notamment quant à l'incidence de ces traitements sur les autres composantes de la microflore du pied des céréales.

Ainsi, VAN DER HOEVEN \& BOLLEN (1980) ont étudié l'action du bénomyl sur le rhizoctone du seigle : au champ, appliqué à fortes doses, ce produit provoque l'augmentation de la maladie tandis qu'in vitro la croissance du champignon est inhibée. REINECKE \& FEHRMANN (1979) quant à eux, ont mis en évidence une corrélation négative entre les évolutions des symptômes dus à $P$. herpotrichoides et de ceux dus à $R$. cerealis ; par contre, BRUCK \& SCHLÖSSER (1982) n'ont pu montrer ce type de corrélation. "L'incompatibilité » apparente in vitro entre $R$. cerealis, d'une part, et $P$. herpotrichoides et Fusarium spp., d'autre part, serait due, selon eux, à l'émission par ces 2 champignons de toxines qui provoqueraient l'inhibition complète de la croissance mycélienne de $R$. cerealis.

L'étude présentée ici se propose de montrer les évolutions comparées de $R$. cerealis et de $P$. herpotrichoides soit indépendamment l'un de l'autre, soit ensemble, en présence ou non d'un traitement au carbendazime. 3 types d'essais ont été réalisés :

- In vitro, sur milieu gélosé pour comparer les sensibilités respectives au carbendazime de $P$. herpotrichoides et de $R$. cerealis,

- sur cultures de blé en terrines placées en conditions semi-contrôlées pour étudier l'influence de différentes méthodes d'infestation et d'un traitement au carbendazime sur l'évolution des 2 champignons,

- en conditions normales de culture enfin, pour suivre l'évolution au champ des 2 agents pathogènes dans des parcelles traitées ou non au carbendazime.

\section{MATÉRIEL ET MÉTHODE}

\section{A. Sensibilité in vitro de $P$. herpotrichoides et de $\boldsymbol{R}$. cerealis au carbendazime}

Le fongicide mis en suspension dans de l'eau stérile est incorporé dans du milieu gélosé à 2 p. 100 d'extrait de malt maintenu en surfusion à $50^{\circ} \mathrm{C}$. Le mélange homogénéisé est distribué dans des boîtes de Petri de $9 \mathrm{~cm}$ de diamètre $(20 \mathrm{ml}$ par boîte). Les con- centrations finales de carbendazime sont en progression géométrique de raison 2 , allant de $0,03 \mu \mathrm{g} / \mathrm{ml}$ à $16 \mu \mathrm{g} / \mathrm{ml}$. Après refroidissement, des explantats calibrés (6,5 $\mathrm{mm}$ de diamètre) prélevés au front de croissance d'une jeune colonie mycélienne sont déposés au centre des boîtes qui sont placées ensuite à $20^{\circ} \mathrm{C}$ à l'obscurité. Les diamètres des plages mycéliennes sont mesurés au bout de $5 \mathrm{j}$ pour $R$. cerealis, $10 \mathrm{j}$ pour $P$. herpotrichoides dont la croissance est plus lente. On estime les concentrations en carbendazime qui inhibent la vitesse de croissance de 50 p. 100 (C I 50).

\section{B. Evolution des deux maladies en conditions semi- contrôlées (serre plastique)}

\section{En l'absence de traitement fongicide}

Cet essai nous a permis de comparer différentes méthodes et différentes dates d'apport de l'inoculum.

\section{a) Isolats utilisés - Production de l'inoculum}

Dans tous les cas, l'isolat de $R$. cerealis utilisé est le $\mathrm{n}^{\circ} \mathrm{RC} 2$ de notre mycothèque; celui de $P$. herpotrichoides provient d'un isolement sur blé effectué l'année des essais.

L'inoculum de $R$. cerealis est produit sur grains d'orge humidifiés par une quantité d'eau égale en poids et stérilisés par 2 passages à l'autoclave $(1 \mathrm{~h}$ à $120^{\circ} \mathrm{C}$ ), à $24 \mathrm{~h}$ d'intervalle. Ce milieu, une fois ensemencé, est mis en incubation, 3 semaines à $22^{\circ} \mathrm{C}$. L'inoculum de $P$. herpotrichoides est produit selon la même technique mais sur grains d'avoine et avec une durée d'incubation de 8 semaines. mination

b) Dispositif expérimental - Techniques de conta-

Cette expérimentation est effectuée sur des plantes de 2 variétés («Talent » et «Arminda ») cultivées en terrines $(30 \times 30 \times 7 \mathrm{~cm})$ contenant un mélange de terre, sable de rivière et tourbe en volumes égaux. Chaque terrine ( = parcelle élémentaire) reçoit 50 caryopses. L'essai comprend 4 répétitions et est placé sous serre plastique avec chauffage d'appoint pour éviter les températures inférieures à $0{ }^{\circ} \mathrm{C}$.

L'infestation est réalisée selon les modalités suivantes :

\section{- Pour R. cerealis}

= dépôt, au semis, d'un grain d'orge colonisé à proximité immédiate de chaque caryopse de blé,

$=$ dépôt des grains d'orge en surface, au moment $\mathrm{du}$ semis, à raison de $10 \mathrm{~g} / \mathrm{m}^{2}$,

= dépôt des grains d'orge, en surface, au stade 2 feuilles de la plante à raison, respectivement, de 10 et $20 \mathrm{~g} / \mathrm{m}^{2}$.

- Pour $P$. herpotrichoides, les grains d'avoine sont déposés en surface, au stade 2 feuilles du blé à raison de $10 \mathrm{~g} / \mathrm{m}^{2}$.

Les combinaisons de ces différentes méthodes d'infestation apparaissent dans le tableau 2 .

\section{c) Notations}

Une notation effectuée 5 mois après le semis permet de calculer le pourcentage de plantes présentant des nécroses sur tiges de chacune des 2 maladies. 


\section{Avec traitement fongicide au carbendazime}

\section{a) Production de l'inoculum}

$R$. cerealis (isolat RC2) est cultivé en boîte de Roux sur vermiculite additionnée, en volume égal, d'eau enrichie d'extrait de malt (2 p. 100). L'inoculum est utilisé après 3 semaines d'incubation à $22{ }^{\circ} \mathrm{C}$.

$P$. herpotrichoides est produit sur grains d'avoine humidifiés et stérilisés. L'incubation est de 8 semaines à $22{ }^{\circ} \mathrm{C}$.

\section{b) Dispositif expérimental - Modalités}

L'essai est réalisé sous serre plastique avec les variétés «Lutin », " Rescler », « Roazon », et « Talent». Les plantes sont cultivées en terrines $(30 \times 30 \times 7 \mathrm{~cm})$ contenant un mélange de tourbe et sable dans les proportions respectives de 1/3-2/3. Le dispositif adopté est un « split plot » à 4 répétitions, la parcelle élémentaire étant une terrine de 50 plantes.

Trois modalités d'infestation sont comparées $(R$. cerealis seul, $P$. herpotrichoides seul, $R$. cerealis $+P$. herpotrichoides) à un témoin non infesté. L'inoculum de $R$. cerealis est incorporé, avant le semis, au mélange de culture à la dose de 1 pour 40 en volumes. L'infestation avec $P$. herpotrichoides se fait au stade 2 feuilles de la plante à raison de $10 \mathrm{~g} / \mathrm{m}^{2}$ d'inoculum.

Une partie des terrines reçoit un traitement au carbendazime ( $40 \mathrm{mg} \mathrm{m.a.} / \mathrm{m}^{2}$ ) au stade début montaison du blé, l'autre non.

\section{c) Notations}

Cinq mois après le semis, le pourcentage de plantes présentant des nécroses sur tiges dues à l'une ou l'autre des 2 maladies est déterminé.

\section{Evolution comparée au champ de $R$. cerealis et $P$. herpotrichoides}

\section{a) Isolats utilisés - Production de l'inoculum}

$R$. cerealis (isolat $\mathrm{RC} 2$ ) et $P$. herpotrichoides (isolat de l'année) sont multipliés l'un sur grains d'orge, l'autre sur grains d'avoine.

\section{b) Dispositif expérimental - Modalités}

Le dispositif adopté est un bloc de Fisher répété 4 fois. Chaque parcelle élémentaire a une surface de $12 \mathrm{~m}^{2}$. Le semis (330 grains $/ \mathrm{m}^{2}$, var. «Talent ») a été précoce (13 octobre 1981) afin de favoriser le développement des maladies étudiées. Les parcelles sont infestées avec l'un ou l'autre des champignons, avec les dêux en mélange ou ne sont pas infestées. Dans chaque cas, une parcelle est traitée au carbendazime, l'autre non.

Il existe différentes techniques d'infestation permettant d'obtenir une bonne expression des symptômes des deux maladies considérées; pour des raisons de commodité, au champ, nous avons choisi d'infester les parcelles lorsque le blé atteint le stade 2 feuilles, à raison de $10 \mathrm{~g}$ d'inoculum $/ \mathrm{m}^{2}$ pour chacun des 2 parasites.

En se basant sur l'évolution du piétin-verse dans les parcelles non traitées, 2 traitements au carbendazime (200 g m.a./ha) ont été décidés : l'un au stade 6 (23 mars 1982), l'autre au stade 7-8 (21 avril 1982) du blé (échelle de Feekes).

\section{c) Notations}

Des notations sont effectuées toutes les semaines du 31 mars au 21 mai inclus, puis tous les $15 \mathrm{j}$, les 9 et 26 juin, sur 25 plantes par parcelle, prélevées suivant une diagonale ; les observations portent sur le nombre de brins-maîtres présentant sur gaines ou sur tiges des symptômes de rhizoctone ou de piétin-verse. En cas d'attaque sur tige, celle-ci est coupée transversalement pour apprécier l'importance de la pénétration, mesurée en pourcentage de section nécrosée.

\section{RÉSULTATS}

\section{A. Sensibilité in vitro de $P$. herpotrichoides et} $R$. cerealis au carbendazime

Les CI 50 du carbendazime, estimées sur 16 isolats de $R$. cerealis et 7 isolats de $P$. herpotrichoides, sont regroupées dans le tableau 1 . Si la croissance de $P$. herpotrichoides est inhibée à de très faibles concentra-

\section{TABLEAU 1}

CI 50 du carbendazime sur 16 isolats de $\mathrm{R}$. cerealis $(R C)$ et 7 isolats de $\mathrm{P}$, herpotrichoides $(P H)$

$E D 50$ values for carbendazim on 16 isolates of $\mathrm{R}$. cerealis (RC) and 7 isolates of $\mathrm{P}$. herpotrichoides $(\mathrm{PH})$.

\begin{tabular}{|c|c|c|c|}
\hline \multirow{2}{*}{ Isolat } & \multicolumn{2}{|c|}{ Origine de l'isolat } & \multirow{2}{*}{$\begin{array}{l}\text { CI } 50 \\
\mu \mathrm{g} / \mathrm{ml}\end{array}$} \\
\hline & variété & département & \\
\hline \multicolumn{4}{|c|}{$R$. cerealis } \\
\hline $\mathrm{RC} 16$ & $?$ & 77 Seine-et-Marne & 1,3 \\
\hline RC 10 & Lutin & 77 Seine-et-Marne & 1,4 \\
\hline $\mathrm{RC} 6$ & Lutin & 51 Marne & 1,6 \\
\hline RC 8 & Roazon & 21 Côte-d'Or & 1,7 \\
\hline RC 7 & Hardi & 63 Puy-de-Dôme & 1,7 \\
\hline RC 3 & Talent & 02 Aisne & 1,8 \\
\hline $\mathrm{RC} 2$ & Roazon & 36 Indre & 1,9 \\
\hline $\mathrm{RC} 5$ & Top & 35 Ille-et-Vilaine & 1,9 \\
\hline RC 15 & $?$ & 71 Saône-et-Loire & 1,9 \\
\hline RC 9 & Top & 86 Vienne & 1,9 \\
\hline $\mathrm{RC} 1$ & $?$ & 29 Finistère & 2,0 \\
\hline $\mathrm{RC} 4$ & Corin & 51 Marne & 2,0 \\
\hline $\mathrm{RC} 10$ & Lutin & 77 Seine-et-Marne & 2,1 \\
\hline $\mathrm{RC} 11$ & Arminda & 80 Somme & 2,1 \\
\hline $\mathrm{RC} 12$ & $?$ & 60 Oise & 2,5 \\
\hline $\mathrm{RC} 14$ & $\mathrm{H} 80 \mathrm{R} 25$ & 35 Ille-et-Vilaine & 3,2 \\
\hline \multicolumn{4}{|c|}{$P$. herpotrichoides } \\
\hline PH 6 & Fidel & 28 Eure-et-Loir & 0,04 \\
\hline PH 7 & Cocagne & 21 Côte-d'Or & 0,04 \\
\hline PH 2 & Corin & 10 Aube & 0,05 \\
\hline PH 3 & Adam & 86 Vienne & 0,06 \\
\hline PH 4 & $?$ & 78 Yvelines & 0,06 \\
\hline PH 5 & Arminda & 35 Ille-et-Vilaine & 0,07 \\
\hline $\mathrm{PH} 1$ & Talent & 35 Ille-et-Vilaine & 0,08 \\
\hline
\end{tabular}


tions en fongicide $(0,04$ à $0,08 \mu \mathrm{g} / \mathrm{ml})$, les isolats de $R$. cerealis présentent une relative sensibilité, variable toutefois suivant les isolats $(1,3 \mu \mathrm{g} / \mathrm{ml}<\mathrm{CI} 50<$ $3,2 \mu \mathrm{g} / \mathrm{ml}$ ).

\section{B. Evolution du piétin-verse et du rhizoctone en con- ditions semi-contrôlées}

\section{En l'absence de traitement fongicide}

Lorsque l'infestation est réalisée avec $R$. cerealis seul et avec l'isolat utilisé, il n'apparaît pas de différences significatives entre les comportements des 2 variétés « Talent » et « Arminda » (tabl. 2). Il n'y a pas de différences non plus entre les différentes méthodes d'infestation. Par contre, dans tous les cas d'infestation avec les 2 champignons, on observe une réduction sensible du pourcentage de plantes présentant des nécroses dues à $R$. cerealis; dans ces conditions, la variété "Arminda » est moins attaquée que «Talent », particulièrement dans le cas d'infestations tardives de $R$. cerealis (stade 2 feuilles).

\section{Après traitement au carbendazime}

Les analyses des notations exprimées en pourcentage de plantes atteintes par l'une ou l'autre des maladies montrent des différences significatives entre variétés (tabl. 3).

Ainsi, "Roazon », tolérant vis-à-vis du piétinverse, apparaît plus sensible au rhizoctone que "Talent » et «Rescler ». En fait, l'examen du tableau montre qu'en ce qui concerne la fréquence du rhizoctone, les différences entre variétés sont les plus importantes, soit lorsque l'on traite au carbendazime, soit lorsque l'infestation est réalisée avec les 2 champignons. Il est difficile dans ces conditions de faire la part entre l'effet du traitement (direct sur $R$. cerealis ? indirect par $P$. herpotrichoides ?) et le niveau de sensibilité de la variété.

On peut observer ainsi que, dans les terrines infestées avec les 2 champignons, le traitement au carbendazime semble avoir permis une meilleure extériorisation du rhizoctone (70 p. 100 au lieu de 45 p. 100 pour «Lutin », 37 p. 100 au lieu de 16 p. 100 pour «Rescler » et 80 p. 100 au lieu de 69 p. 100 pour "Roazon »). Le même effet peut être constaté, dans les terrines infestées avec $P$. herpotrichoides seul, pour les 2 variétés plus sensibles que sont « Lutin » et “Roazon ». Seul "Talent» présente un comportement différent qui se traduit, au niveau de l'analyse statistique de l'ensemble de l'essai toutes variétés confondues, par une différence non significative entre l'intensité des symptômes de rhizoctone observés dans les terrines traitées et non traitées (tabl. 4). Il pourrait s'agir là d'un artefact d'expérimentation si l'on considère que ce comportement de "Talent» ne se confirme pas au champ (fig. 3).

Enfin, la fréquence observée des symptômes de rhizoctone est moindre lorsque $P$. herpotrichoides est introduit en plus de $R$. cerealis (tabl. 4) et ceci bien que le traitement au carbendazime ait été très efficace sur le piétin-verse (tabl. 3).

\section{Evolution comparée, au champ, de $R$. cerealis et $P$. herpotrichoides}

\section{Dans les parcelles non traitées}

Dans les parcelles où $R$. cerealis est apporté seul, ce parasite évolue rapidement du 7 au 21 avril. Le pourcentage de tiges attaquées atteint 68 , correspondant à une section nécrosée de 17 p. 100 (fig. 1).

Dans les parcelles infestées avec $P$. herpotrichoides seul, le piétin-verse évolue régulièrement jusqu'au 14 avril, puis reste à un niveau très élevé : nous avons

TABLEAU 2

Pourcentages de plantes, cultivées en terrines (blé, var. «Talent» et «Arminda »), présentant des nécroses sur tiges dues à R. cerealis et/ou $\mathrm{P}$. herpotrichoides en fonction des dates et doses d'infestations par ces 2 champignons (moyennes de 4 répétitions). Les valeurs moyennes suivies de lettres différentes sont significativement différentes $\grave{a} p=0,05$

Percent of plants grown in pots (wheat cv 'Talent' and 'Arminda') with symptoms on the stems caused by $\mathrm{R}$, cerealis and/or $\mathrm{P}$. herpotrichoides relative to plant stage at inoculation and inoculum density (means of 4 replicates). (Values followed by different letters are significantly different for $p=0.05$.)

\begin{tabular}{|c|c|c|c|c|c|c|c|}
\hline \multicolumn{4}{|c|}{ Modalités d'infestation } & \multicolumn{4}{|c|}{ Pourcentage moyen de plantes présentant des nécroses } \\
\hline \multicolumn{2}{|c|}{$\begin{array}{l}\text { avec } R \text {. cerealis } \\
\text { (grains d'orge) }\end{array}$} & \multicolumn{2}{|c|}{$\begin{array}{l}\text { avec } P \text {. herpotrichoides } \\
\text { (grains d'avoine) }\end{array}$} & \multicolumn{2}{|c|}{ Variété « Talent» } & \multicolumn{2}{|c|}{ Variété « Arminda » } \\
\hline Stade & Dose & Stade & Dose & $\begin{array}{l}\text { dues à } R \text {. } \\
\text { cerealis }\end{array}$ & $\begin{array}{l}\text { dues à } P \text {. her- } \\
\text { potrichoides }\end{array}$ & $\begin{array}{l}\text { dues à } R . \\
\text { cerealis }\end{array}$ & $\begin{array}{c}\text { dues à } P \text {. her- } \\
\text { potrichoides }\end{array}$ \\
\hline semis & $\begin{array}{l}1 \text { grain d'orge } \\
\text { par semence }\end{array}$ & & 0 & $100 \mathrm{a}$ & ld & $68 \mathrm{~b}$ & $8 \mathrm{c}$ \\
\hline semis & $10 \mathrm{~g} / \mathrm{m}^{2}$ & & 0 & $98 a b$ & $9 \mathrm{c}$ & $87 a b$ & $2 c$ \\
\hline 2 feuilles & $10 \mathrm{~g} / \mathrm{m}^{2}$ & & 0 & $95 \mathrm{ab}$ & $16 \mathrm{c}$ & $93 a$ & $8 c$ \\
\hline 2 feuilles & $20 \mathrm{~g} / \mathrm{m}^{2}$ & & 0 & $93 \mathrm{~b}$ & $8 \mathrm{c}$ & $75 \mathrm{ab}$ & $41 b$ \\
\hline & 0 & 2 feuilles & $10 \mathrm{~g} / \mathrm{m}^{2}$ & $1 \mathrm{~d}$ & $100 a$ & od & $100 \mathrm{a}$ \\
\hline semis & $\begin{array}{l}1 \text { grain d'orge } \\
\text { par semence }\end{array}$ & 2 feuilles & $10 \mathrm{~g} / \mathrm{m}^{2}$ & $80 \mathrm{c}$ & $83 b$ & $83 \mathrm{ab}$ & $94 a$ \\
\hline semis & $10 \mathrm{~g} / \mathrm{m}^{2}$ & 2 feuilles & $10 \mathrm{~g} / \mathrm{m}^{2}$ & $83 c$ & $86 \mathrm{~b}$ & $77 \mathrm{ab}$ & $95 a$ \\
\hline 2 feuilles & $10 \mathrm{~g} / \mathrm{m}^{2}$ & 2 feuilles & $10 \mathrm{~g} / \mathrm{m}^{2}$ & $71 \mathrm{c}$ & $95 \mathrm{ab}$ & $38 \mathrm{c}$ & $100 \mathrm{a}$ \\
\hline 2 feuilles & $20 \mathrm{~g} / \mathrm{m}^{2}$ & 2 feuilles & $10 \mathrm{~g} / \mathrm{m}^{2}$ & $77 \mathrm{c}$ & $96 a b$ & $36 c$ & $99 a$ \\
\hline
\end{tabular}


TABLEAU 3

Pourcentages de plantes (blé var. « Roazon », « Talent», "Rescler », "Lutin ») cultivées en terrines et présentant des symptômes sur tiges, 5 mois après infestation par $\mathrm{R}$. cerealis et/ou $\mathrm{P}$. herpotrichoides. Les plantes de certaines terrines sont traitées au carbendazime $140 \mathrm{mg}$ m.a. $/ \mathrm{m}^{2}$ ) au stade montaison du blé (moyennes de 4 répétitions). Les valeurs moyennes suivies de lettres différentes sont significativement différentes à $p=0,05$.

Percent of plants (wheat cv 'Roazon', 'Talent', 'Rescler', 'Lutin') grown in pots with eyespot and/or sharp eyespot symptoms, 5 months after inoculation with R. cerealis and/or P. herpotrichoides. Plants of some pots have been treated with carbendazim $\left(40 \mathrm{mg} / \mathrm{a} . \mathrm{i} . / \mathrm{m} \mathrm{m}^{2}\right) \mathrm{during}$ stem extension (means of 4 replicates). Values followed by different letters are significantly different for $p=0.05$.

\begin{tabular}{|c|c|c|c|c|c|c|c|c|c|}
\hline \multirow{3}{*}{ Infestation } & \multirow{3}{*}{$\begin{array}{c}\text { Traitement } \\
\text { au } \\
\text { carbendazime } \\
40 \mathrm{mg} \mathrm{m} \text {.a. } / \mathrm{m}^{2}\end{array}$} & \multicolumn{8}{|c|}{ Pourcentage moyen de plantes présentant des nécroses } \\
\hline & & & dues à & cerealis & & & es à $P$. & otrichoide & \\
\hline & & Roazon & Talent & Rescler & Lutin & Roazon & Talent & Rescler & Lutin \\
\hline Non infesté & non & 3 & 3 & 9 & 14 & 0 & 3 & 3 & 4 \\
\hline \multirow{2}{*}{ avec $R$. cerealis } & non & 82 & 84 & 62 & 75 & 0 & 0 & 0 & 0 \\
\hline & oui & 84 & 59 & 68 & 82 & 0 & 0 & 0 & 0 \\
\hline \multirow[b]{2}{*}{ avec $P$. herpotrichoides } & non & 3 & 0 & 2 & 1 & 27 & 77 & 69 & 81 \\
\hline & oui & 26 & 7 & 4 & 23 & 0 & 4 & 0 & 3 \\
\hline \multirow{3}{*}{$\begin{array}{l}\text { avec } R . \text { cerealis } \\
\quad+ \\
P .\end{array}$} & non & 69 & 45 & 16 & 45 & 24 & 63 & 89 & 70 \\
\hline & oui & 80 & 30 & 37 & 70 & 0 & 0 & 0 & 0 \\
\hline & $\begin{array}{l}\text { Valeur } \\
\text { moyenne }\end{array}$ & $49,6 b$ & $32,6 \mathrm{a}$ & $28,3 \mathrm{a}$ & $44,3 a b$ & $7,3 \alpha$ & $21 \beta$ & $23 \beta$ & $22,6 \beta$ \\
\hline
\end{tabular}

noté une attaque sur tiges de 95 p. 100 correspondant à un pourcentage de section nécrosée de 72 (fig. 2).

Lorsque les infestations ont été effectuées avec les 2 champignons simultanément, les attaques de piétinverse sur tiges atteignent 93 p. 100, par contre celles de rhizoctone ne dépassent pas $38 \mathrm{p} .100$, correspondant à des pourcentages respectifs de section nécrosée de 65 et de 12 (fig. 3).

\section{TABLEAU 4}

Pourcentage de plantes (blé - toutes variétés confondues : "Roazon 》, «Talent », « Rescler », "Lutin »), cultivées en terrines et présentant des nécroses dues à $\mathrm{R}$. cerealis, en fonction du type d'infestation et avec ou sans traitement au carbendazime. Les valeurs suivies de lettres différentes sont significativement differentes pour $p=0,05$.

Percent of plants (wheat - all cultivars 'Roazon', 'Talent', 'Rescler', 'Lutin') grown in pots, with sharp eyespot depending on inoculations and carbendazim treatment. Values followed by different letters are significantly different for $p=0.05$.

\begin{tabular}{lcc}
\hline \hline Infestation & $\begin{array}{c}\text { Traitement } \\
\text { au carbendazime } \\
40 \mathrm{mg} \mathrm{m.a.} / \mathrm{m}^{2}\end{array}$ & $\begin{array}{c}\text { Fréquence de } \\
\text { nécroses dues à } \\
\text { transformation } \\
\text { angulaire }\end{array}$ \\
\hline avec $R$. cerealis & non & $61,82 \mathrm{e}$ \\
\cline { 2 - 3 } & oui & $60,12 \mathrm{de}$ \\
\hline avec $P$. herpotrichoides & non & $3,79 \mathrm{a}$ \\
\hline $\begin{array}{l}\text { avec } P \text {. herpotrichoides } \\
\text { et } R \text {. cerealis }\end{array}$ & oui & $19,98 \mathrm{~b}$ \\
\cline { 2 - 3 } & non & $39,66 \mathrm{c}$ \\
\hline
\end{tabular}

\section{Dans les parcelles traitées}

Dans les parcelles infestées avec $R$. cerealis seul, il n'y a pas de différence entre les parcelles traitées et non traitées (fig. 1). Dans celles infestées avec $P$. herpotrichoides seul, le pourcentage de tiges attaquées atteint 62 (20 en ce qui concerne le pourcentage de section nécrosée) (fig. 2).

Dans les parcelles où nous avons réalisé une infestation simultanée avec $R$. cerealis et $P$. herpotrichoides (fig. 3), le pourcentage de tiges attaquées par ce dernier ne dépasse pas 56 p. 100 , avec 20 p. 100 de section nécrosée. Par contre, en ce qui concerne $R$. cerealis le pourcentage de tiges atteintes est de 57 (15 pour le pourcentage de section nécrosée). Par rapport aux parcelles non traitées, l'augmentation du rhizoctone est significative quand on considère les p. 100 de tiges attaquées, elle ne l'est pas pour les sections nécrosées.

\section{DISCUSSION - CONCLUSION}

Que ce soit en conditions semi-contrôlées ou dans les parcelles non traitées, au champ, il semble que si l'on assure la présence simultanée, au contact des plantes, d'inoculums primaires de $P$. herpotrichoides et $R$. cerealis, ce dernier ait quelques difficultés à $s$ 'installer alors que l'installation puis la progression de $P$. herpotrichoides ne sont pas gênées. $P$. herpotrichoides serait bien le parasite dominant du complexe parasitaire du pied des céréales.

Malgré l'effet mis en évidence in vitro, aucun de nos essais en terrines ou au champ ne montre d'efficacité de traitement au carbendazime sur $R$. cerealis.

Toutefois, les valeurs obtenues pour les CI 50 mettent en évidence une grande différence de sensibilité 


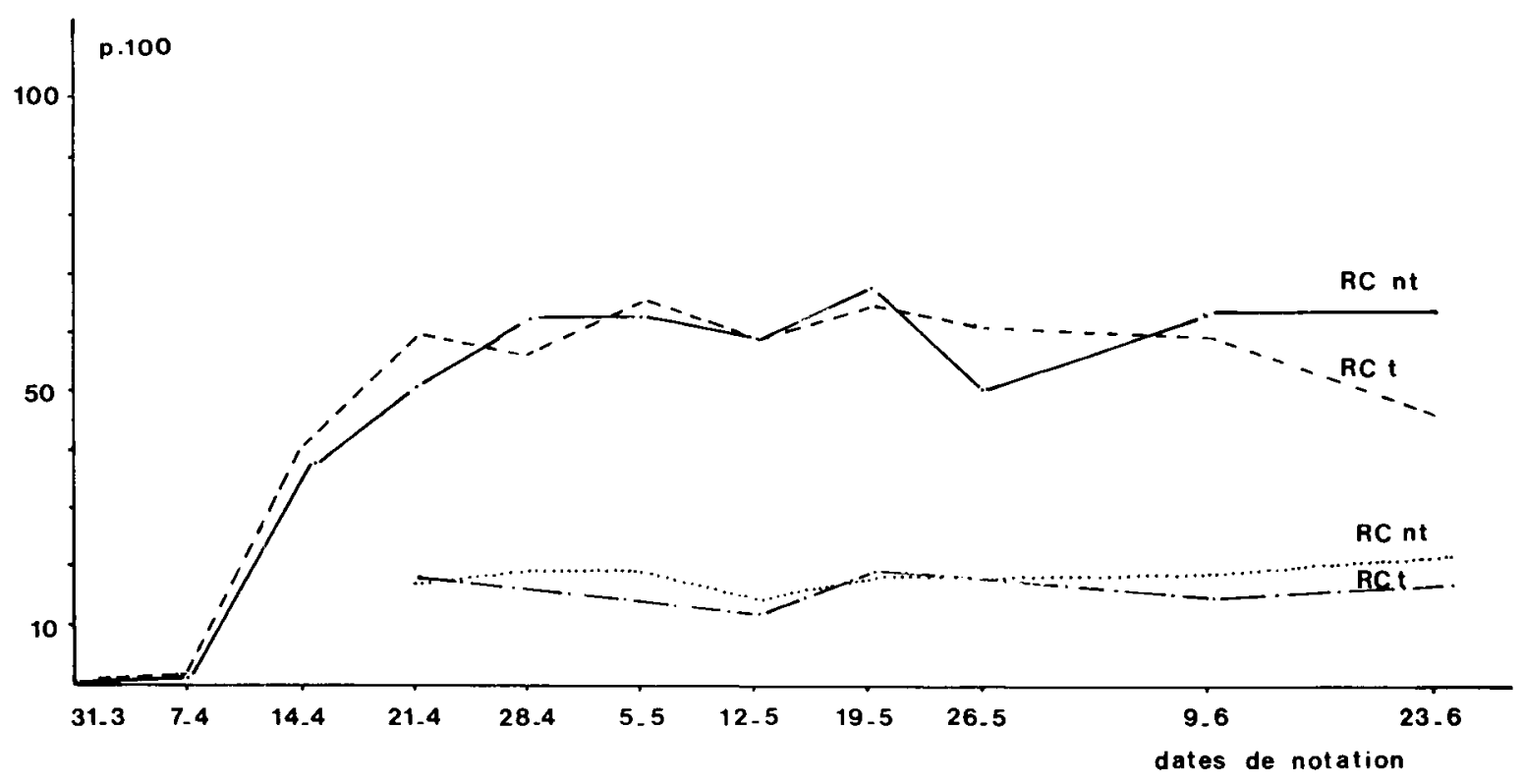

Figure 1

Evolution du pourcentage de tiges attaquées (blé, var. «Talent ») et du pourcentage de section nécrosée par $\mathrm{R}$. cerealis dans les parcelles infestées avec R. cerealis seul $\left(10 \mathrm{~g}\right.$ de grains d'orge colonisés $\left./ \mathrm{m}^{2}\right)$.

----- RCt : Pourcentage moyen de tiges attaquées dans les parcelles traitées au carbendazime $(200 \mathrm{~g} \mathrm{m.a./ha)}$.

RCnt : Pourcentage moyen de tiges attaquées dans les parcelles non traitées.

- - RCt: Pourcentage moyen de section nécrosée dans les parcelles traitées au carbendazime $(200 \mathrm{~g}$ m.a. / ha $)$.

.... RCnt : Pourcentage moyen de section nécrosée dans les parcelles non traitées.
Percent of stem with symptoms caused by R. cerealis and percent of necrotic stem section in wheat $c v$ «Talent " in plots inoculated with R. cerealis $\left(10 \mathrm{~g}\right.$ of inoculum $\left./ \mathrm{m}^{2}\right)$.

- $R C t$ : mean percent of stems with symptoms in carbendazim treated plots. $200 \mathrm{~g}$ a.i./ha.

RCnt : mean percent of stems with symptoms in untreated plots.

-.- RCt mean percent necrosis of stem cross section in carbendazim treated plots (200 g a.i./ha).

..... RCnt: mean percent necrosis of stem cross section in untrated plots.

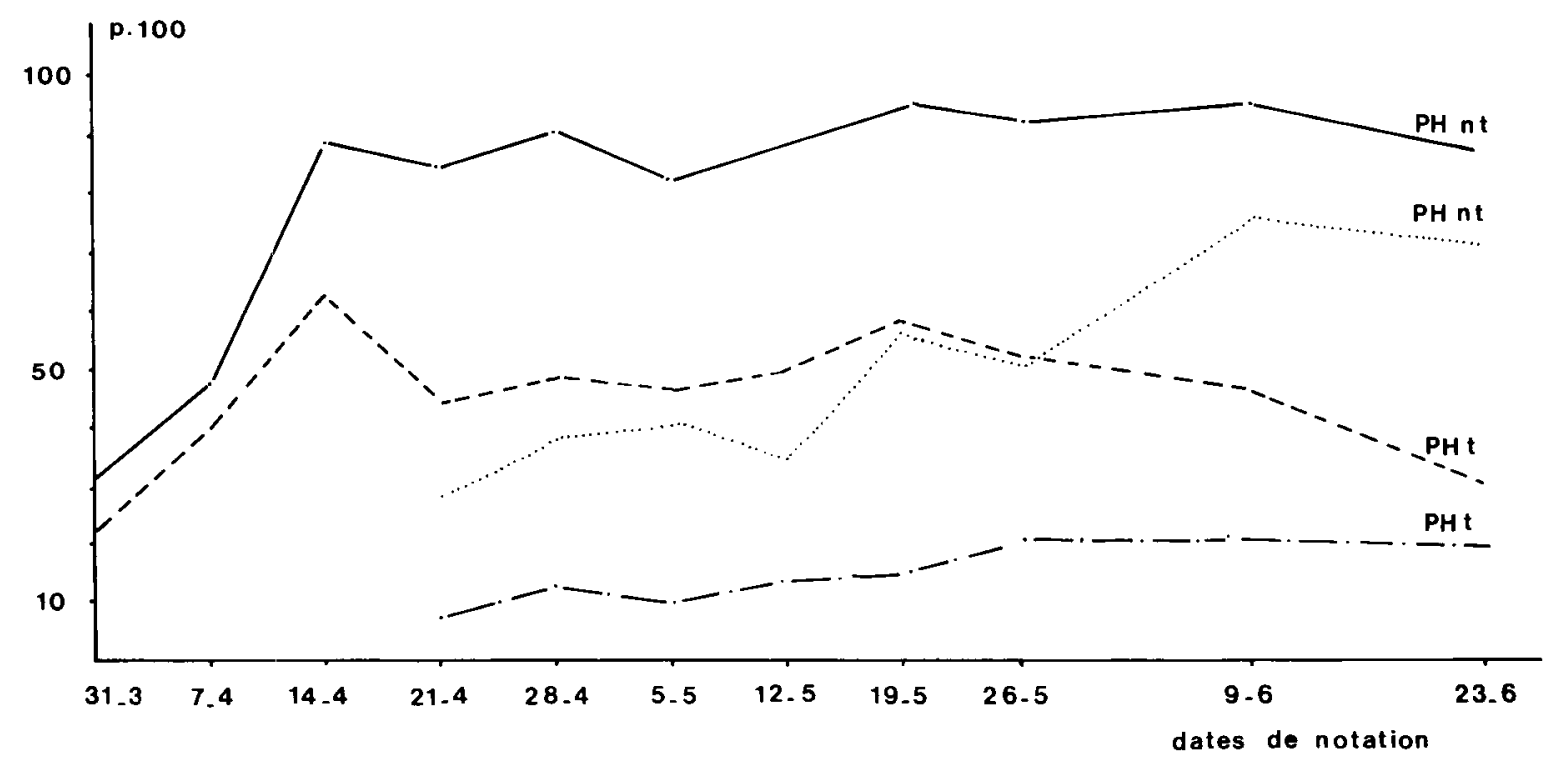

Figure 2

Evolution du pourcentage de tiges attaquées et du pourcentage de section nécrosée par $\mathrm{P}$. herpotrichoides dans les parcelles infestées avec $\mathbf{P}$. herpotrichoides seul ( $10 \mathrm{~g}$ de grains d'avoine colonisés $\left./ \mathrm{m}^{2}\right)$ (blé var. "Talent»).

---- PHt: Pourcentage moyen d'attaques sur tiges dans les parcelles traitées au carbendazime $(200 \mathrm{~g}$ m.a./ha).

PHnt : Pourcentage moyen d'attaques sur tiges dans les parcelles non traitées.

-.- PHt : Pourcentage moyen de section nécrosée dans les parcelles traitées au carbendazime $(200 \mathrm{~g}$ m.a./ha).

..... PHnt : Pourcentage moyen de section nécrosée dans les parcelles non traitées.
Percent of stems with symptoms and percent of necrotic stem sections caused by P. herpotrichoides in plots inoculated with this pathogen ( $10 \mathrm{~g}$ of inoculum $\left./ \mathrm{m}^{2}\right)$.

PHt : Mean percent of stems with symptoms in carbendazim treated plots (200 g a.i./ha). plots.

PHnt : Mean percent of stems with symptoms in untreated

- - PHt : Mean percent necrosis of stem cross section in carbendazim treated plots (200 g a.i./ha).

..... PHnt: Mean percent necrosis of stem cross section in untreated plots. 

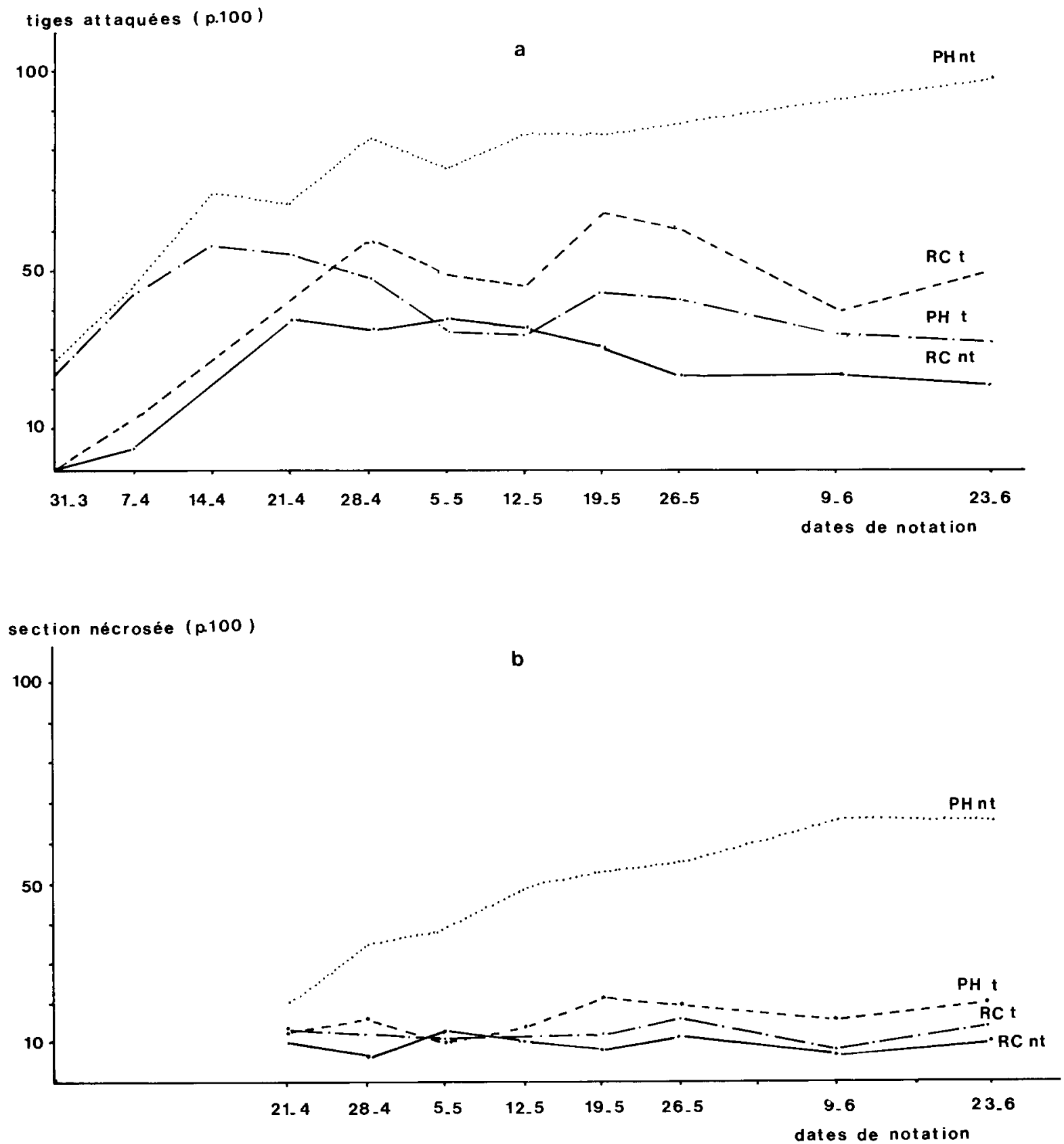

Figure 3

Evolution au champ du pourcentage de tiges attaquées (fig. $3 a$ ) et du pourcentage de section nécrosée (fig. $3 b$ ) par $\mathrm{R}$. cerealis $(R C)$ et par $\mathrm{P}$. herpotrichoides $(\mathrm{PH})$ dans les parcelles infestées simultanément par ces 2 champignons et traitées au carbendazime ( $t$ ) (200 g m.a./ha) ou non traitées (nt). Blé variété «Talent».

entre les 2 parasites. Ces différences pourraient avoir une importance sur le développement des 2 maladies dans une culture. Il est possible que, compte tenu de la localisation sur la plante de ces 2 maladies, la quantité de produit qui arrive en contact avec $R$. cerealis ne soit pas suffisante.

Quand le piétin-verse disparaît ou reste à un niveau d'intensité très bas, le rhizoctone se développe. Nous avons constaté au champ une augmentation significative de $R$. cerealis dans les parcelles traitées au carbendazime. Cependant, au niveau de la section nécrosée, cette augmentation n'est pas sensible. Ceci semble correspondre au fait que $R$. cerealis a un développe-
Percent of stems with symptoms (fig. $3 a$ ) and percent necrosis of stem cross section (fig. 3 b) caused by R. cerealis (RC) and by $\mathrm{P}$. herpotrichoides $(P H)$ in plots of wheat $c v$ «Talent $"$ simultaneously inoculated with these fungi and treated with carbendazim (200 g a.i./ha) (t) or not (nt).

ment dans la tige beaucoup plus lent que $P$. herpotrichoides. Nos résultats vont dans le sens des travaux de REINECKE \& FEHRMANN (1979) et de VAN DER HOEVEN \& BOLLEN (1980) sur le seigle. Il est possible que la suppression d'un des composants de la microflore, dans notre cas $P$. herpotrichoides, augmente les possibilités d'infection par un autre pathogène. Cette action directe dont le mécanisme reste à élucider, n'est sans doute pas la seule. On connaît l'efficacité des benzimidazoles sur certains champignons comme les Trichoderma (DAVET, 1981 ; BAICU, 1982) par ailleurs antagonistes de $R$. cerealis (NIGNON, 1982, non publié). Une étude spécifique du développement de 
$R$. cerealis en relation avec d'autres microorganismes du pied des céréales serait nécessaire.

On peut se poser également la question de l'opportunité d'utiliser des fongicides à systémie ascendante contre les maladies du pied. Il faut que la quantité de produit nécessaire soit présente à la base de la plante, à l'intérieur des gaines, au moment opportun. Nous nous proposons d'étudier, en relation avec l'évolution des parasites, la persistance et le devenir dans la plante de différentes matières actives, qu'elles soient systémiques, pénétrantes ou de surface.
Les résultats obtenus dans cette étude le sont à partir d'un seul isolat de $R$. cerealis mais, compte-tenu de la variabilité existant au sein de cette espèce (LUCAS \& CAVElier, 1983), il serait bon de suivre l'évolution du complexe $P$. herpotrichoides - $R$. cerealis avec différents isolats de $R$. cerealis représentatifs de cette variabilité.

\section{RÉFÉRENCES BIBLIOGRAPHIQUES}

Baicu T., 1982. Toxicity of some pesticides to Trichoderma viride Pers., Crop Protection, 1, 349-358.

Bruck K. P., Schlösser E., 1982. Getreidefusskrankheitserreger. V. Antagonismus zwischen den Erregern. Z. Pflanzenkr. Pflanzenschutz, 89 (6) 337-343.

Davet P., 1981. Effets de quelques pesticides sur la colonisation d'un substrat par le Trichoderma harzianum Rifai en présence des autres champignons du sol. Soil Biol. Biochem., 13, 513-517.

Defosse L., 1970. Essais de lutte chimique contre le piétin-verse, Cercosporella herpotrichoides Fron, avec un fongicide systémique. Parasitica, 26, 3-14.

Doussinault G., 1970. Problèmes posés par l'amélioration de la résistance du blé tendre vis-à-vis du piétin-verse (Cercosporella herpotrichoides Fron). Ann. Amélior. Plantes, 20 (4), 433-452.

Fehrmann H., 1970. Bekämpfung der Halmbruchkrankheit des Weizens mit Benomyl. Nachrichtenbl. dtsch. Pflanzenschutzdienstes (Stuttgart), 22, 136-139.

Glynne M. B., 1936. Some new British records of fungi on wheat, Cercosporella herpotrichoides Fron, Gibellina cerealis Pass. and Ophiobolus herpotrichus (Fr.) Sacc. Trans. Br. Mycol. Soc., 20 (2) 120.
Lemaire J. M., Doussinault G., Jouan B., 1970. Nouvelle perspective de lutte contre le piétin-verse des céréales par traitement des semences. C. R. Acad. Agric. Fr., 56, 643-646.

Lucas Ph., Cavelier N., 1983. Rhizoctonia cerealis Van der Hoeven, agent du rhizoctone des céréales en France. Caractéristiques et variabilité. Agronomie, 3 (9), 831-838.

Ponchet J., 1959. La maladie du piétin-verse des céréales : Cercosporella herpotrichoides Fron. Importance agronomique, biologie, épiphytologie. Ann. Epiphyt., 10, 46-99.

Rapilly F., 1970. La détermination des dates de traitements fongicides appliqués par voie aérienne sur céréales en végétation, cas du blé d'hiver. Phytiatr. Phytopharm., 19, 185-203.

Reinecke P., Fehrmann H., 1979. Rhizoctonia cerealis Van der Hoeven an Getreide in der Bundesrepublik Deutschland. Z. Pflanzenkr. Pflanzenschutz, 86 (3/4), 190-204.

Sprague R., 1931. Cercosporella herpotrichoides Fron, cause of the Columbia basin foot rot of winter wheat. Science N.S., 74, 51-53.

Van der Hoeven E. P., Bollen G. J., 1980. Effects of benomyl on soil fungi associated with rye. I. Effects on the incidence of sharp eyespot caused by Rhizoctonia cerealis. Neth. J. Plant Pathol., 86, 163-180. 